\title{
Light scattering of glass-particle filled matrices with similar refractive index
}

\section{Wolfgang Wildner (1) and Dietmar Drummer}

\begin{abstract}
If fillers can be added to transparent materials without losing transparency, then advantages like enhanced mechanical and thermal properties can be integrated. The investigated specimens consist of glass particles and refractive index oil as a model for transparent matrices with a very similar refractive index. Their optical properties and resulting limitations are described. Potential uses are also demonstrated by application-oriented optical testing. Besides a standard spectrometer, additional spectrometer setups were used. These include a diffuse as well as a collimated illumination and different sample positioning. Furthermore, the scattered light intensity was measured at different angles. This analysis reveals that composites with smaller particles transmit more light directly. In contrast, standard spectrometers indicate an increasing direct transmittance of composites with larger particles. They collect significant amounts of scattered light and, therefore, are not suitable for transmission measurements of such composites. The different positioning shows that all specimens exhibit very little scattering when placed directly on a diffuse light source. With a greater distance between specimen and light source, the scattering increases strongly. To display the composites' optical appearance, the lightdark-contrast of the diffuse white light source photographed behind the composite was analyzed. Both long and short distances between composite and light source lead to a precise image of the light source. Nevertheless, the white light source appears in the color of the wavelength with matching refractive indices at long distances.
\end{abstract}

\section{Keywords}

Glass particles, optical compound, light scattering

\section{Introduction}

Glass particle filled systems with similar refractive indices (RIs) between fillers and matrix have promising properties for the use in transparent and optical components. Glass particles and beads in a polymer matrix lead to an increased stiffness ${ }^{1-3}$ and a reduced thermal expansion., ${ }^{4,5}$ Theoretically, transparency is not impaired when the RIs of the materials perfectly match. ${ }^{6}$ In addition to the goal of producing glass-filled parts with unimpaired transmission and enhanced properties, the scattering between matrix and glass particles can be used for various purposes. For example, the temperature-dependent RI of polymers in combination with the almost temperature-independent optical properties of glass particles causes a temperaturedependent transmission. This enables the material combination for fiber-optic temperature sensing. ${ }^{7,8}$

For both, the production of transparent composites and the use of their specific transmission properties, it is important to describe and measure their optical properties. In a theoretical approach, the light scattering of small spherical particles can be calculated with Mie's Theory. ${ }^{9}$ The transmitted intensity $I_{t}$ of an incident light $I_{0}$ is given by

$$
I_{t}=I_{0} \exp (-A z)
$$

where $z$ is optical path length and $A$ is the attenuation coefficient which is expressed as the following

$$
A=n C_{a}+n C_{s}
$$

where $n$ is the number of particles per unit volume and $C_{a}$ and $C_{s}$ are the absorption cross section and

Institute of Polymer Technology, Friedrich-Alexander-University Erlangen-Nürnberg (FAU), Germany

\section{Corresponding author:}

Wolfgang Wildner, Institute of Polymer Technology, Am Weichslegarten 9, Erlangen 91052, Germany.

Email: wildner@lkt.uni-erlangen.de 
scattering cross section. For multiple different nonabsorbing particles, the attenuation coefficient can be written as

$$
A=\sum n_{j} C_{s j}
$$

where $n_{j}$ is the number per volume unit of a specific particle $j$ and $C_{s j}$ is its scattering cross section. The calculation of the scattering cross section is described in many books, i.e. van de Hulst ${ }^{9}$ and Meeten ${ }^{10}$ and depends on the RI ratio $m$, among others

$$
m(\lambda)=\frac{n_{\text {Particle }}(\lambda)}{n_{\text {Matrix }}(\lambda)}
$$

where $n_{\text {Particle }}$ and $n_{\text {matrix }}$ are the RIs of the particles and the matrix, respectively.

As simplifying assumptions, multiple scattering effects are ignored and the RIs of particles and the matrix are presumed as isotropic and real (not absorbing particles). Nevertheless, Groever et al. ${ }^{11}$ show, among others, that the calculation with the Mie Theory does not lead to accurate results for a composite with irregularly shaped glass particles whose geometry is far from an ideal sphere. The calculated transmission exceeds the measured values. The reasons stated by the authors for the mismatch are near-field effects and irregularities of the particles. ${ }^{11}$ Kahnert et al. ${ }^{12}$ describe computed results of the Muellermatrix for various individual particles, including spherical particles with randomly rough surfaces. It was not possible to identify any simple pattern for the influence of the surface roughness on the scattering intensity. Additionally, these computations are very elaborate and time-consuming. Muinonen et al. ${ }^{13}$ calculated the scattering of large sand particles $\left(r_{\text {eff }}=125 \mu \mathrm{m}\right)$ using ray optics. The light scattering by Gaussian-randomrough particles also was computed by Zubko et al. ${ }^{14}$ As a result, roughness manly affects the polarization and backscattering, but there is no general influence of particle roughness on scattering properties.

In general, there is scarce information about the optical properties of multiple rough particles with an RI similar to the matrix. First experiments about the transmission of these composites were carried out by Christiansen. ${ }^{15,16} \mathrm{He}$ recognized that these materials could be used to produce monochromatic light. Its wavelength corresponds to the wavelength at the intersection of the materials' RIs. The group of Kagawa conducted numerous experiments on the light scattering of glass-filled, transparent polymers. ${ }^{4,5,17-20}$ They present an empirical model for transmission calculations based on the law of Lambert-Beer and the particle surface area. ${ }^{17}$ As a result, small particles scatter more and transmit less light, and spectrometer measurements confirm these results. The given reason for this is that the small particles have a larger particlematrix interface. Dunlap and Howe ${ }^{21}$ describe the calculation using the Rayleigh-Gans-Debye (RGD) approximation, a simplification ${ }^{22}$ of Mie's theory under certain conditions. ${ }^{9}$ They include empirical modifications to describe interparticle interference and multiple scattering. Thus, they reproduce the scattering of oil/glass mixtures with a high volume fraction of glass in good agreement with the measurement results. Research on the transmission of glass fiber-filled transparent polymers was done by the Stoffer group. ${ }^{23-27}$ They describe a model for the temperature dependent transmission of fiber reinforced poly(methyl methacrylate) also using to Mie's Theory. ${ }^{23}$ They show a good agreement between calculation and measurement for $17 \mu \mathrm{m}$ fibers. They also highlight the bad influence on the transmission of nonwet fibers. Their model indicates a higher transmission for fibers with a smaller diameter, but they do not provide measurements of different fiber diameters.

Standard spectrometers are usually used to measure the transmission properties. In these measurements, the transmitted light is usually defined as the light exiting the specimen within a defined angle, for instance, $\pm 2.5^{\circ} .{ }^{10}$ Therefore, scattered light in small angles is added to the transmitted intensity. ${ }^{21}$ As a result, the transmission of particles ${ }^{4,17}$ and fibers ${ }^{24,28}$ with a larger diameter exceeds the transmission of smaller fillers. Only Dunlap and Howe ${ }^{21}$ address the fact, that the aperture of the transmission detector significantly influences the measurement result.

When designing components made of reinforced, transparent compounds, the visual appearance of an object behind is decisive. It strongly depends on the positioning of the object, the composite and the spectator. ${ }^{10}$ These influences are not considered in standard spectrometer measurements. In this work, we present the analysis of the scattering in relation to wavelength, RI difference, light source, and positioning of components. Specimens consisting of glass particles with different sizes and with a similar RI to the matrix were used for these measurements. In addition to the parameters mentioned above, the angle-dependent scattering intensity was analyzed. Additionally, the transmission properties were evaluated with respect to the image contrast of the pictured light source.

\section{Significance of the research}

The practical relevance of the topic becomes clear when considering the patents and patent applications dealing with a material combination of glass particles and transparent plastics in order to create a transparent composite. ${ }^{29-40}$ The combination of transparency with reduced 
thermal expansion ${ }^{29-35,37}$ and better mechanical properties $^{36,38,40}$ is usually given as the main reason for development. These properties open up a wide range of possible applications. Often mentioned is a material for transparent overmoulding or sealing of light-emitting diodes (LEDs) and optoelectronic components, ${ }^{30-35,37}$ but also for transparent plates and glazings ${ }^{30,37,38,40}$ and generally for optical and transparent components. ${ }^{30,37,38}$ In order to maintain the transparency of the composite material, a maximum RI difference is usually specified. Values in a wide range from $0.01^{29,30,34,37-39}$ to $0.001^{32,33}$ for a maximum allowable RI difference are given. What is missing; however, as in the scientific publications, is a differentiated consideration of the influences of various factors on the optical properties under practical conditions of use. This knowledge is necessary for the use of such a material combination.

\section{Methodology}

\section{Materials and specimen}

For the investigations, RI oil with an RI of $n_{D}=1.5700$ (Cargille Labs) was selected as the matrix along with glass particles (N-BAK4, Schott) with an RI $n_{D}=$ 1.56883 and a density $\rho=3.05 \mathrm{~g} / \mathrm{cm}^{3}$. The glass particles were produced by milling of bulk glass. The milling was done with the planetary micro mill Pulverisette 7 classic line (Fritsch $\mathrm{GmbH}$ ). Grinding bowls in the size of $45 \mathrm{ml}$ and grinding balls, both made of zirconia, were operated at $600 \mathrm{r} / \mathrm{min}$. The minimum grinding time was $3 \mathrm{~min}$. Subsequently, the particles were screened with laboratory sieves with mesh widths of $28 \mu \mathrm{m}, 56 \mu \mathrm{m}, 90 \mu \mathrm{m}$, and $125 \mu \mathrm{m}$ to get three size fractions. To reduced small dust particles, the particles were ultrasonically washed in distilled water and then dried. Figure 1 shows scanning electron microscope (SEM) images of the particles. Due to the manufacturing process, they have an irregular shape and rough surfaces.

In order to prepare the specimens, the glass particles were poured together with the RI oil into glass cuvettes
(Schott) with a path length $z$ of $1.0 \mathrm{~mm}$ and $2.0 \mathrm{~mm}$. To analyze the transmission with the spectrometer of a path length $z$ of $3.0 \mathrm{~mm}$, the 1.0 and $2.0 \mathrm{~mm}$ cuvettes were assembled with a thin oil film to reduce surface reflections. The filling degree of the specimen was measured by weighing the cuvettes with and without glass. The total thickness of the cuvettes is $3 \mathrm{~mm}$ for the cuvette with a path length of $1 \mathrm{~mm}$ and $4 \mathrm{~mm}$ for the cuvette with a path length of $2 \mathrm{~mm}$.

\section{Particle size}

The volumetric particle size distribution was optically analyzed with the instrument Morphologi (Malvern Instruments). With this method, the particles were dispersed on a glass plate. Each particle was photographed with a microscope and the equivalent diameter was calculated. Two measurements were made for each particle size fraction and at least 100,000 particles were evaluated per measurement.

\section{Optical analysis}

The wavelength dependent RI was analyzed with a standard Abbe refractometer (Kruess AR 2008). Optical bandpass filters (EdmundOptics, wavelengths of $450 \mathrm{~nm}, 500 \mathrm{~nm}, 550 \mathrm{~nm}, 600 \mathrm{~nm}$, and $650 \mathrm{~nm}$ ) were attached to the light source in order to measure the RI at different wavelengths. The RI at each wavelength was calculated as follows.

Figure 2 shows the refraction of light at the refractometer's prism. With the refractive angle $\gamma$ of grazing light, the prism angle $\Psi$ of $62^{\circ},{ }^{41}$ the RI of the prism at $589.3 \mathrm{~nm} n_{\text {Prism (D) }}$ made of the glass N-SF4 (Schott), the displayed RI of the refractometer $n_{\text {Display }}$, the RI of air $n_{\text {air }}=1$, and Snellius' law, ${ }^{42}$ the angle of refraction $\delta$ at the prism was calculated

$$
\frac{n_{\text {Display }}}{n_{\text {Prism }(D)}}=\frac{\sin \gamma}{\sin \frac{\pi}{2}}=\sin \gamma
$$
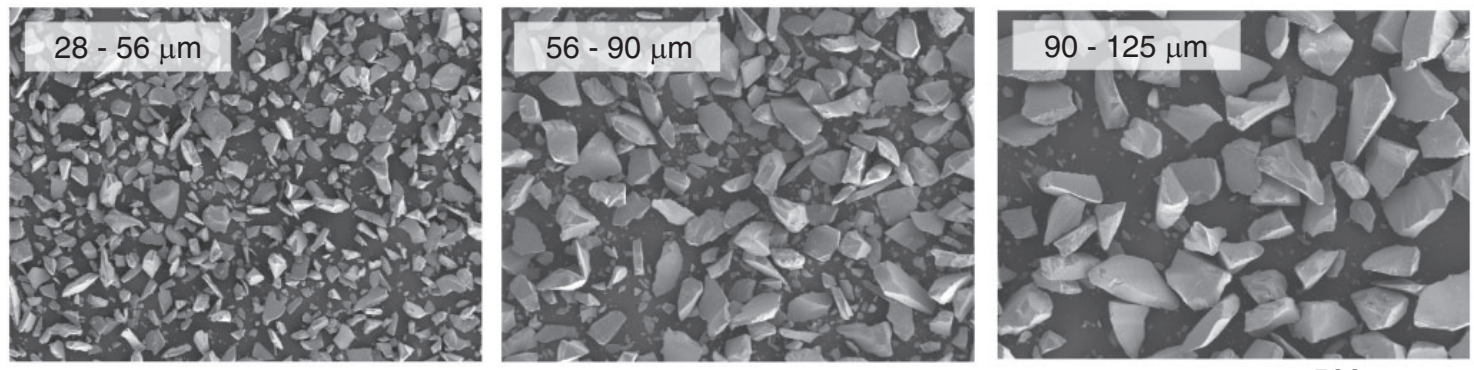

$500 \mu \mathrm{m}$

Figure I. SEM images of the particles in three different size sections. 
$\frac{n_{\text {Prism }(D)}}{n_{\text {air }}}=\frac{\sin \delta}{\sin \beta}=\frac{\sin \delta}{\sin (\psi-\gamma)}=\frac{\sin \delta}{\sin \psi \cos \gamma-\cos \psi \sin \gamma}$

Equations (5) and (6) lead to $\delta$

$$
\begin{aligned}
\sin \delta & =n_{\text {Prism }(D)} \sin \psi \cos \gamma-n_{\text {Display }} \cos \psi= \\
& =n_{\text {Prism }(D)} \sin \psi \sqrt{1-\sin ^{2} \gamma}-n_{\text {Display }} \cos \psi= \\
& =n_{\text {Prism (D) }} \sin \psi \sqrt{1-\frac{n_{\text {Display }}^{2}}{n_{\text {Prism }(D)^{2}}}}-n_{\text {Display }} \cos \psi= \\
& =\sin \psi \sqrt{n_{\text {Prism }(D)}^{2}-n_{\text {Display }}^{2}}-n_{\text {Display }} \cos \psi
\end{aligned}
$$

Solving equation (7) for $n_{\text {Display }}$ and replacing $n_{\text {Display }}$ and $n_{\text {Prism ( } D)}$ with the wavelength dependent RIs of the

\section{Diffuse monochromatic light}

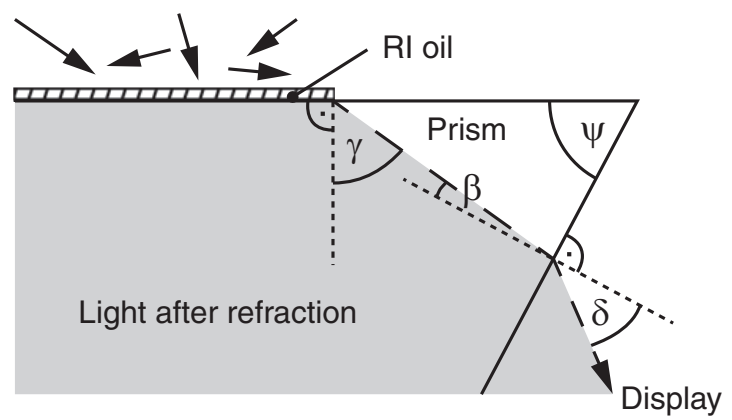

Figure 2. Refraction at the refractometer's prism. oil $n(\lambda)$ and Prism $n_{\operatorname{Prism}(\lambda)}$ leads to equation (8). By using $\sin \delta$ from equation (7), the oil's RI $n(\lambda)$ can be calculated as follows ${ }^{43,44}$

$$
\begin{aligned}
& n(\lambda)=-\cos \psi \sin \delta+\sqrt{\left.(\cos \psi \sin \delta)^{2}+n_{\text {Prism }(\lambda)}\right)^{2} \sin ^{2} \psi-\sin ^{2} \delta} \\
& =\sin \psi \sqrt{n_{P r i s m(\lambda)}^{2}-\sin ^{2 \delta}}-\cos \psi \sin \delta
\end{aligned}
$$

Finally, the oil's RI in the range between $350 \mathrm{~nm}$ and $750 \mathrm{~nm}$ was approximated with Cauchy's Equation ${ }^{45}$

$$
n(\lambda)=A+\frac{B}{\lambda^{2}}+\frac{C}{\lambda^{4}}
$$

where $A, B$, and $C$ are constants and $\lambda$ is the wavelength in $\mathrm{nm}$.

The transmission properties were analyzed with different instruments. The measurement temperature was $25^{\circ} \mathrm{C}$ for all transmission measurements. A standard spectrometer (Lambda 18 by Perkin Elmer Inc.) was applied to analyze the transmission in a range of $350-750 \mathrm{~nm}$ with a gap width of $1 \mathrm{~nm}$. In order to measure the influence of the particles only, the transmission was evaluated in proportion to the transmission of an oil-filled cuvette without glass particles.

A modifiable measurement setup was designed to analyze the transmitted intensity in relation to the light source, positioning, and scattering angle, Figure 3. This spectrometer setup consists of a stabilized halogen light source (SLS201, Thorlabs) which

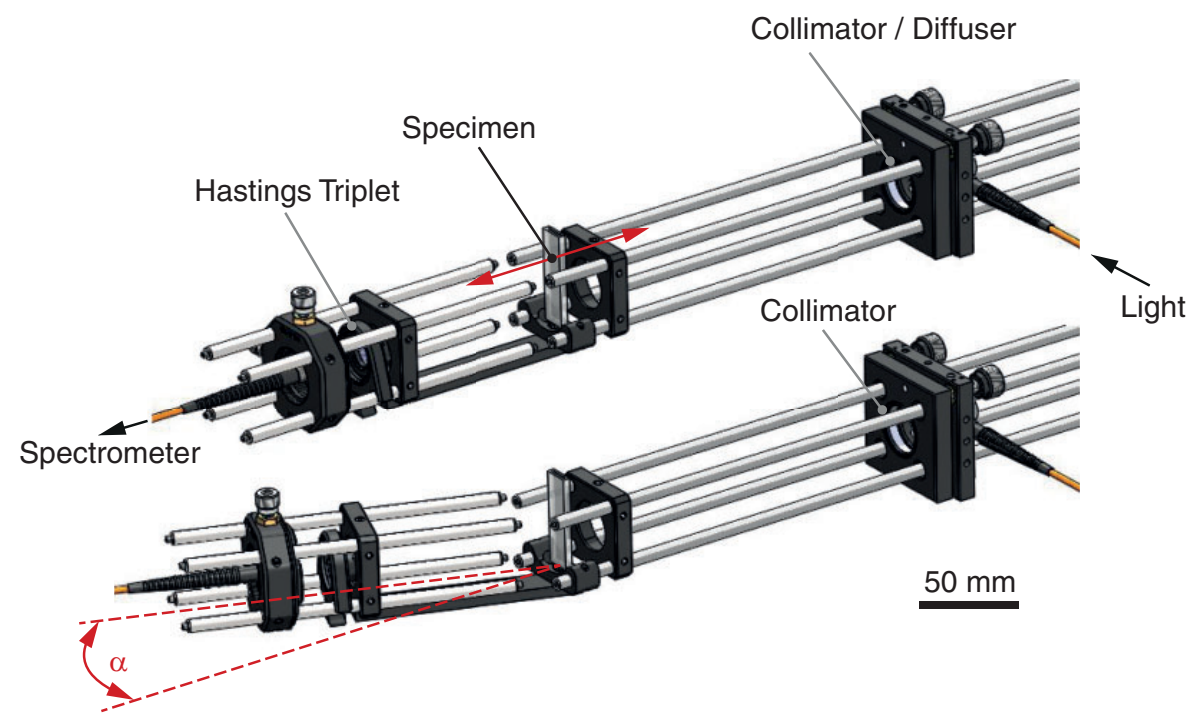

Figure 3. Schematic picture if the measurement setup to evaluate the transmission in relation to the distance and light source (above) and angle (below). 
feeds the light into an optical fiber. At the end of the fiber, the light is decoupled by a reflective collimator (RC02FC-P01, Thorlabs) or a cosine corrector (CCSA1, Thorlabs). Thereby, collimated or diffuse light can be generated.

After passing the specimen, a Hastings Triplet (TRH127-020-A-ML, Thorlabs) focuses the light into another optical fiber with a core diameter of $200 \mu \mathrm{m}$. This fiber is connected to a CCD-Spectrometer (CCS100, Thorlabs). The spectrometer analyzes the intensity of the incoming light. To get results that are independent of the light source's intensity distribution, the results are divided by the recorded intensity without a specimen in the light path. The integration time of the spectrometer can be adjusted to measure in different intensity ranges.

The transmission properties were calculated according to the Mie theory. The scattering cross sections were calculated for the different particle sizes. The free program Mieplot by Philip Laven ${ }^{46}$ was used for these computations. The number size distribution, analyzed with

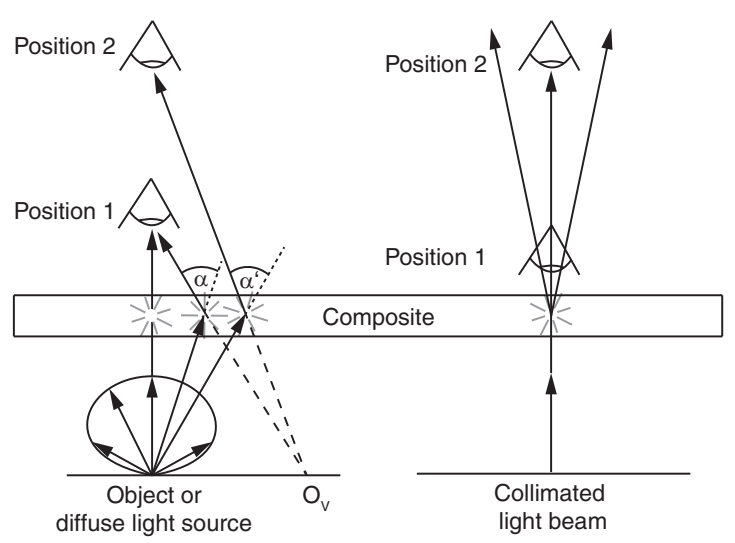

Figure 4. Schematic picture of the scattering of a diffuse light source (left, based on Kahnert et al. ${ }^{12}$ ) and a collimated beam. the instrument Morphologi as described above, served as an input parameter for the particle size.

In order to analyze the scattered intensity in relation to the angle, the focus unit was rotated around the specimen, Figure 3 below.

The transmitted intensity of a scattered collimated beam depends strongly on the distance between the specimen and the analyzer. For this reason, the distance between the specimen and fiber tip leading to the spectrometer was varied using a collimated light beam. Conversely, the transmitted intensity of a diffuse light source alters with the space between the light source and the specimen. Figure 4 further illustrates this: if the spectator observes an object behind a composite, the scattering leads to a blurred picture. It looks as if the object is also positioned in the virtual object point $\mathrm{O}_{\mathrm{V}}$ (Figure 4, left). If the spectator increases its distance from position 1 to position 2, the object's appearance remains almost the same due to the similar scattering angle $\alpha^{\prime}$. However, a decreasing distance between object and composite results in a more precise picture as $\mathrm{O}_{\mathrm{V}}$ approaches the object. On the other hand, the collected light of a collimated beam declines with increasing distance between a spectator and the composite (Figure 4, right: Position 1 to 2).

Figure 5 clarifies the specimen's positioning in the custom spectrometer. Depending on the light source, the distance s between the light source and composite (in the case of a diffuse light source) or between the collecting fiber tip and composite (in the case of a collimated beam) will be given.

The transmitted light was photographed to illustrate the scattering properties of the specimen. A camera (Canon EOS 10D) without an objective lens was placed in the light path at a distance of $900 \mathrm{~mm}$ from the composite in order to take a picture of the transmitted collimated beam. Hereby, the light was photographed directly by the image sensor of the camera. In the case of the diffuse light source, the camera with a mounted

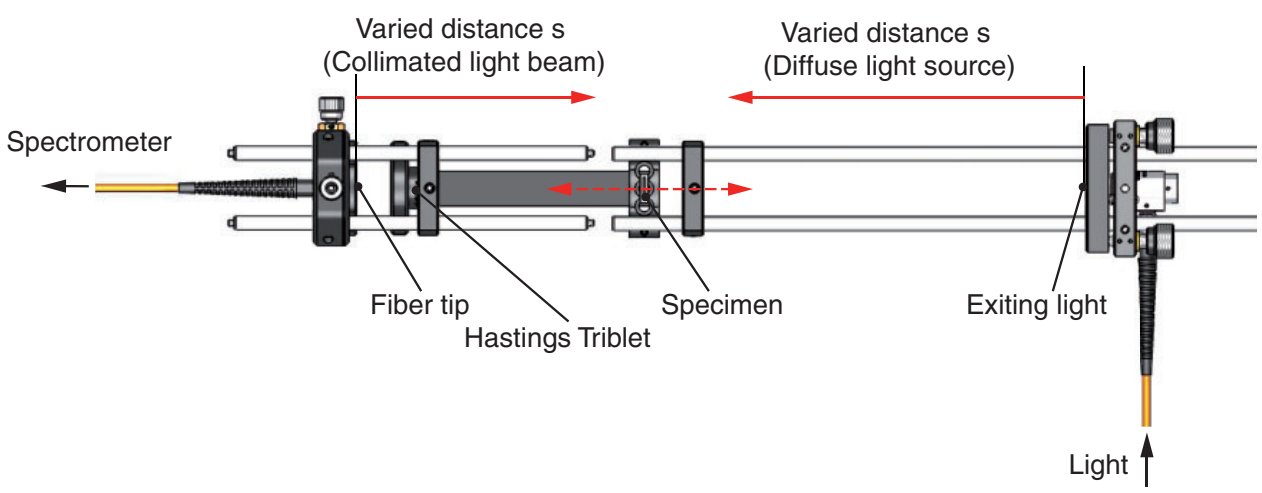

Figure 5. Positioning of the specimen in dependence of the measuring task. 
objective lens was focused on the light source while the specimen was placed in front of the camera. The composite was placed at distances of $0 \mathrm{~mm}, 10 \mathrm{~mm}$, and $220 \mathrm{~mm}$ from the light source. In both cases, the Weber contrast $K_{w}{ }^{47}$ of the pictured light spot in relation to the background was calculated as follows

$$
K_{w}=\frac{\bar{l}(a)}{\bar{l}(b)}-1
$$

where $\bar{l}(a)$ and $\bar{l}(b)$ are the mean luminescence over the lines $a$ and $b$, respectively (Figure 6). The mean luminescence was analyzed at TIFF-images of the photographs and has a value in the range from zero (black) to 255 (white).

\section{Results and discussion}

Figure 7 shows the different particle sizes used to prepare the specimens. The average particle diameters $d_{50.3}$ of the three size sections are $50 \mu \mathrm{m}, 95 \mu \mathrm{m}$, and $150 \mu \mathrm{m}$.

Figure 8 displays the filling degree of the oil and particle filled cuvettes in relation to the particle size. The volume of the large particles exceeds the volume of the small particles about $10 \%$.

Figure 9 shows the RI of the two materials at $25^{\circ} \mathrm{C}$. The materials have a matching RI at about $640 \mathrm{~nm}$. The RI ratio $m$ decreases strongly with lower wavelengths, whereas there are only slight differences between the RIs at wavelengths $>640 \mathrm{~nm}$.

Figure 10 shows the transmission of RI oil in a cuvette with a thickness of $1 \mathrm{~mm}$. There is a strong decline in transmission below a wavelength of $400 \mathrm{~nm}$, due to the absorption characteristics of the RI oil. The loss of about $8 \%$ at larger wavelengths is caused by surface reflections at both sides of the specimen.

Figure 11 shows the transmission, analyzed with the standard spectrometer in relation to wavelength, particle size, and specimen thickness. In order to exclude

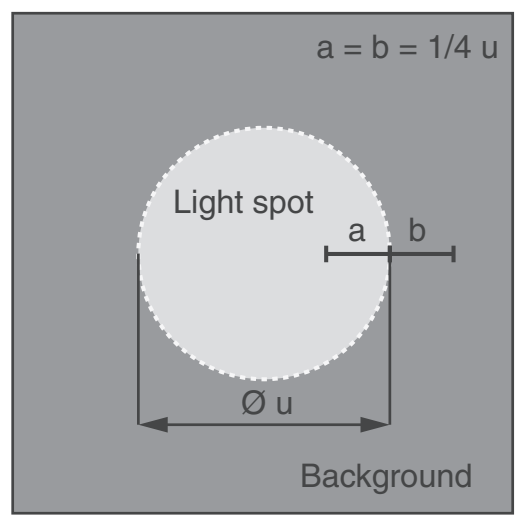

Figure 6. Luminescence analysis for contrast calculations.

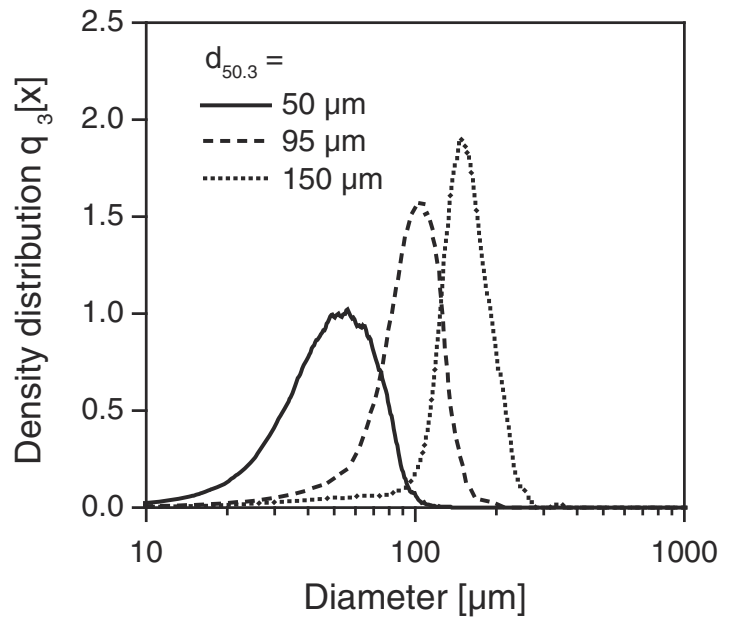

Figure 7. Volumetric particle size distribution of the three sieved size sections.

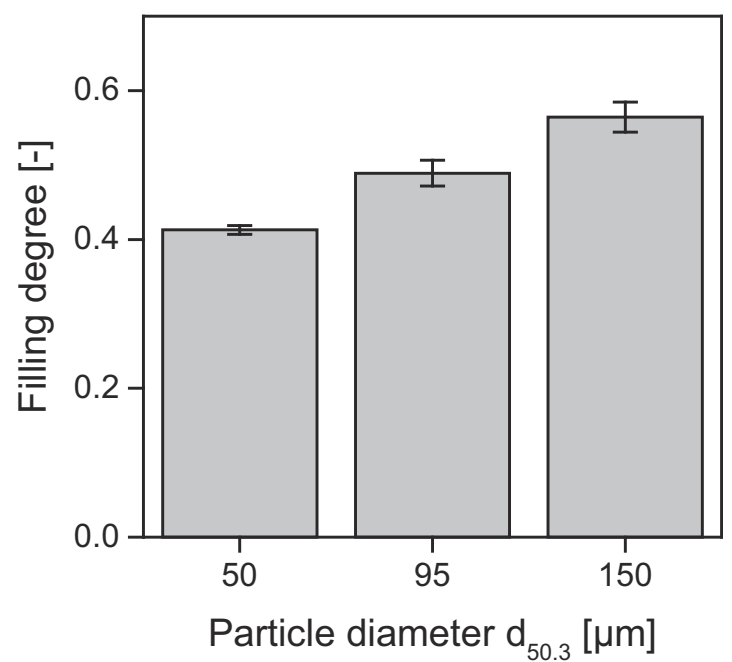

Figure 8. Filling degree in dependence of the particle size.

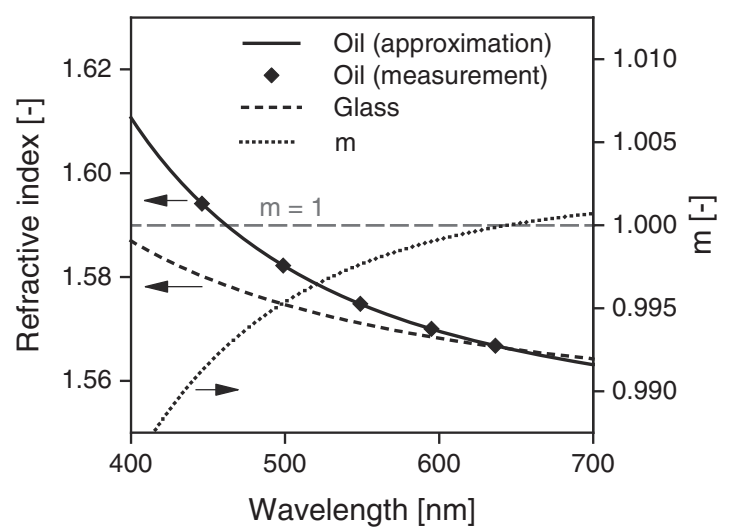

Figure 9. RI of oil, glass, and RI ratio $\mathrm{m}$ in dependence of the wavelength. 
wavelength independent influences such as surface reflections and transmission losses due to remaining imperfections, the curves are further displayed normalized to a maximum transmission of $100 \%$. As described in the introduction section, a smaller particle size and increasing path length $z$ (specimen thickness) results in less transmittance. Moreover, looking at the original curves, the maximum transmission at matching RIs declines slightly with decreasing particle size and increasing thickness. Reasons, therefore, may be inhomogeneities in the RIs or defects in the composite, such as air inclusions and small cracks in the particles or non-wetted particles.

Figure 12 shows the normalized transmission in relation to the RI ratio $m$. It reveals the same transmission characteristic as Figure 11: Decreasing particle size $d$ and increasing thickness $z$ result in a lower transmission at a specific $\mathrm{m}$. Thus, at an RI ratio $\mathrm{m}$ of 0.98 , almost

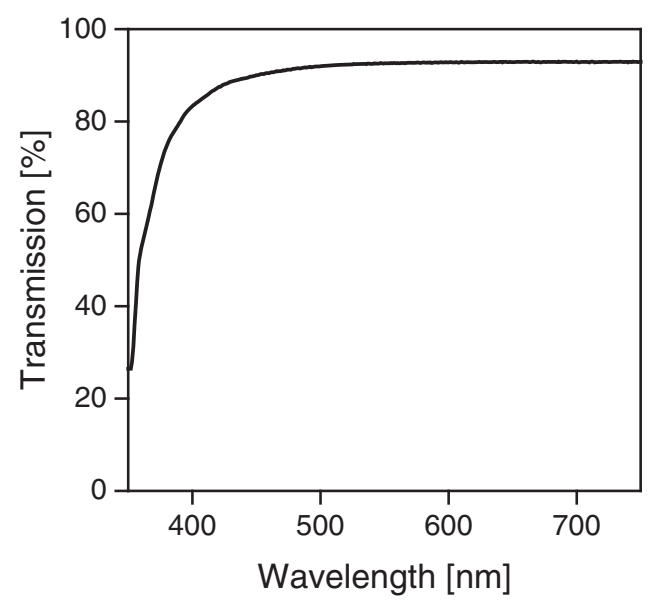

Figure 10. Transmission of RI oil in a cuvette $(z=1 \mathrm{~mm})$. no transmitted light was detected for a thickness $\geq 2 \mathrm{~mm}$ and a particle size $\leq 95 \mu \mathrm{m}$.

Figure 13 shows the standardized transmission of a collimated light beam in relation to the distance $\mathrm{s}$ from the specimen to the fiber tip, which leads to the spectrometer. The measurement was done with the custom spectrometer. The longer the distance, the less scattered light is coupled into the fiber to the spectrometer. Similar to the standard spectrometer measurements, the transmittance curve of thicker specimens is steeper at the equal distances. Noteworthy is the steeper transmission curve at long distances, compared to standard spectrometer measurements. This indicates that scattered light is additionally included to the direct transmittance in standard spectrometer measurements.

The transmission, analyzed with the custom spectrometer, decreases up to a distance of $40 \mathrm{~mm}$ between specimen and fiber tip. At greater distances; however, no additional reduction in transmitted light can be observed. This indicates that from this point the analyzed transmittance equals the real direct transmittance without any shares of scattered light.

At short distances between 0 and $4 \mathrm{~mm}$ larger particles transmit more light, just as observed with the standard spectrometer. Relevant shares of scattered light can reach the collecting fiber at this distance, similar to the sensor of a standard spectrometer. Conversely, at a distance $>4 \mathrm{~mm}$ small particles transmit more light due to their higher direct transmittance. Consequently, the transmittance of composites with large particles decreases faster with a longer distance between the composite and the analyzer.

The results of Figure 13 are summarized in Figure 14 for a maximum RI ratio to achieve a transmission $\geq 90 \%$. Thus, comparatively low values for $\mathrm{m}$ are possible if the composite is in contact with the collecting fiber tip representing the spectator. As illustrated by
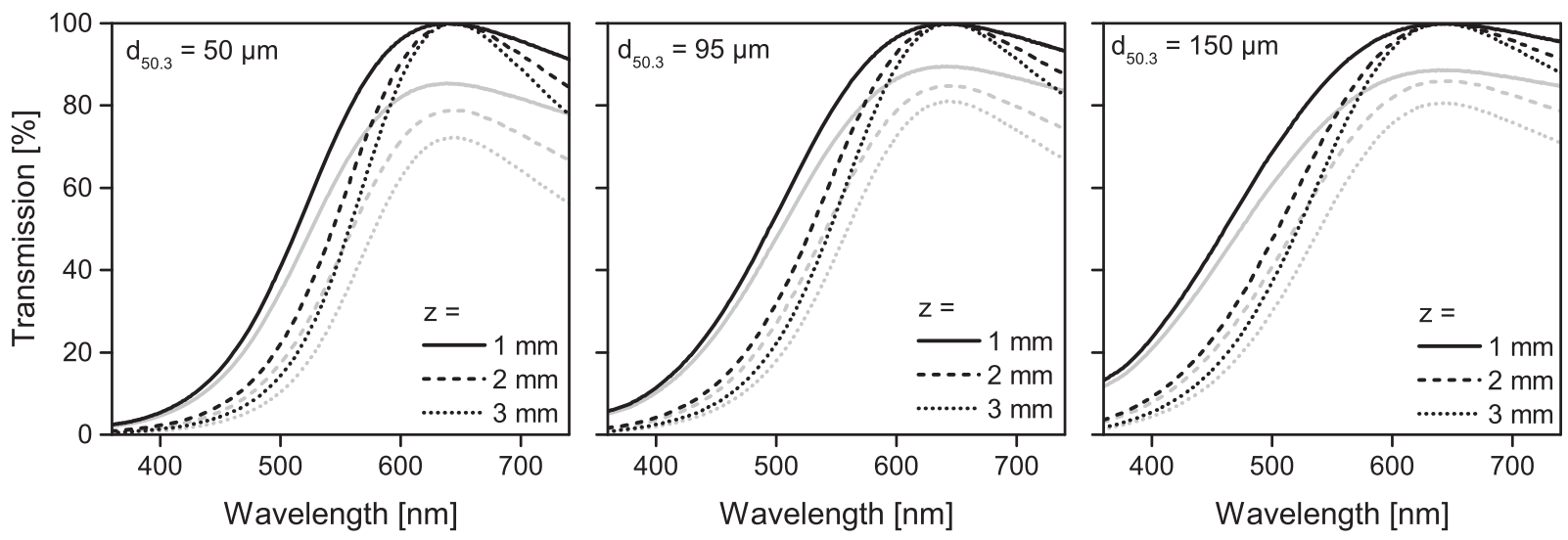

Figure II. Transmission in relation to the wavelength, particle size and specimen thickness: as measured (gray) and normalized (black), analyzed with the standard spectrometer. 

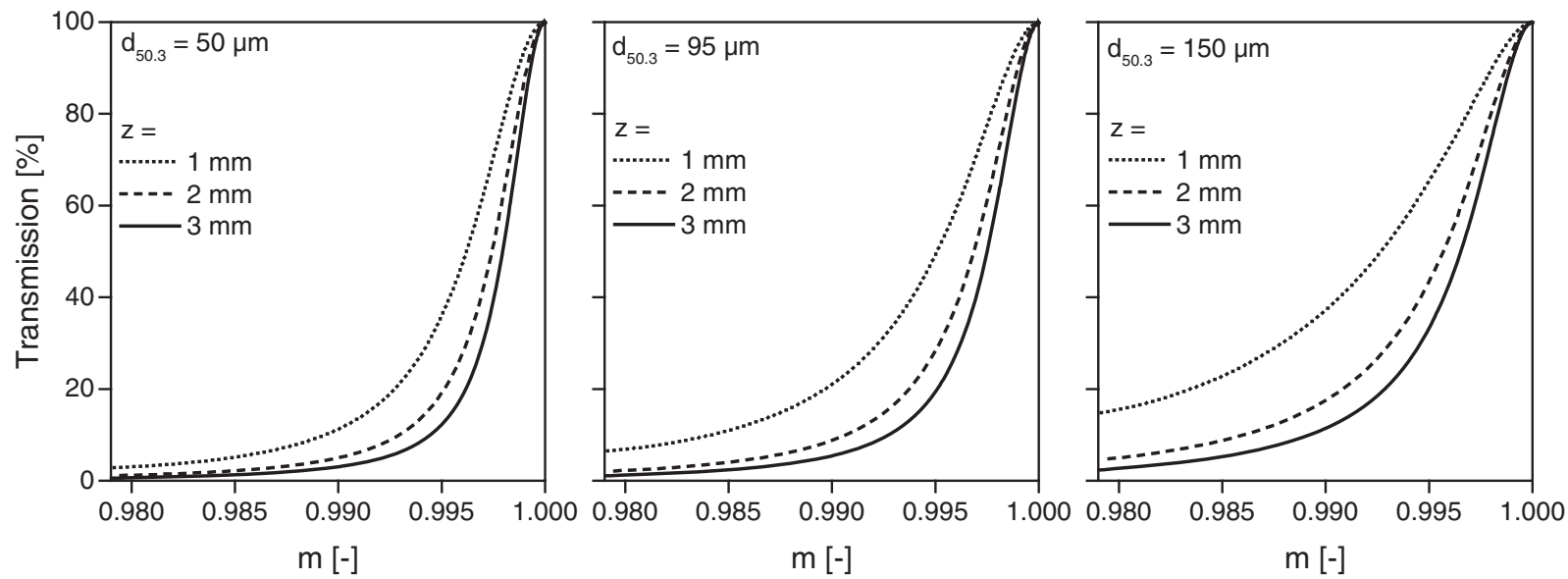

Figure 12. Normalized direct transmittance in relation to $\mathrm{m}$, analyzed with the standard spectrometer.
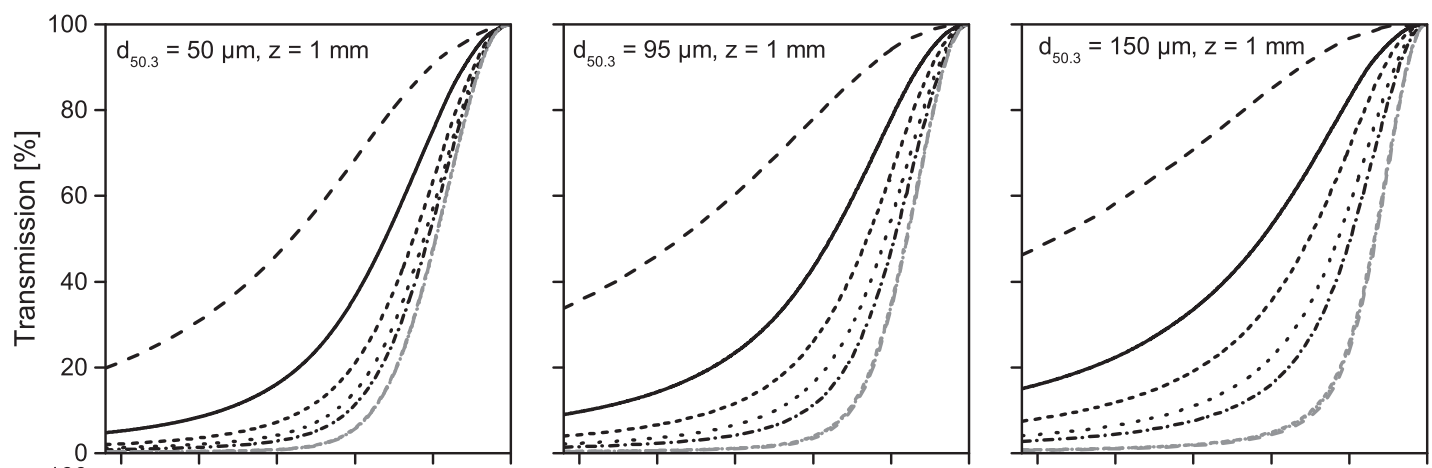

Distance to analyzator: - $-0 \mathrm{~mm}$ $-2 \mathrm{~mm}$ $\cdots 4 \mathrm{~mm}$ $\cdots 6 \mathrm{~mm}$ $-\cdot-\cdot 8 \mathrm{~mm}$ - $-40 \mathrm{~mm}$ -... $160 \mathrm{~mm}$
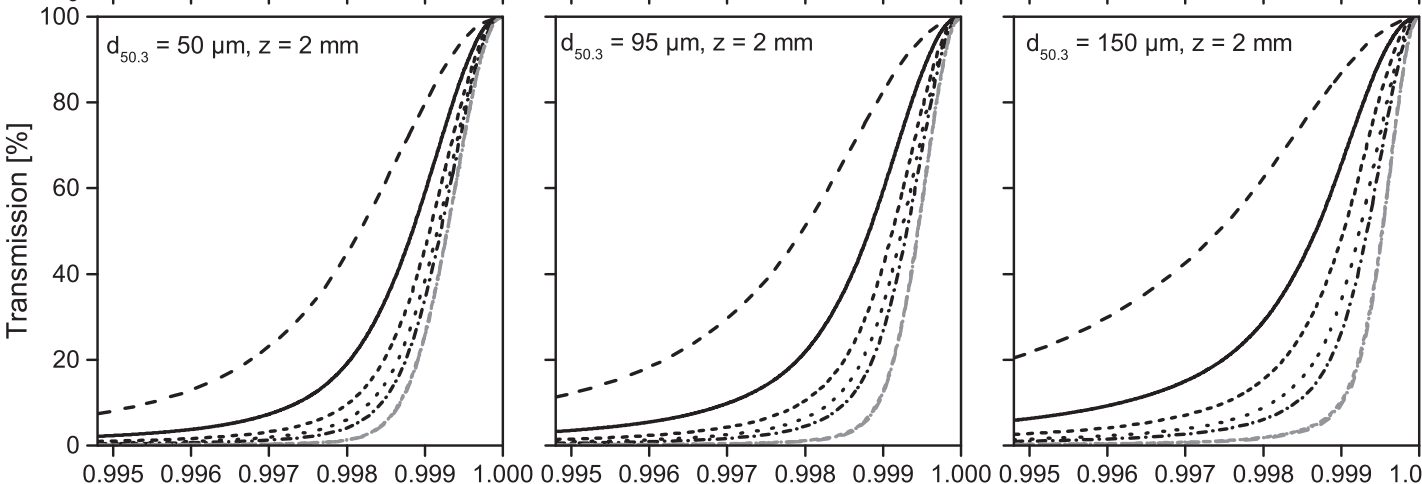

$\mathrm{m}[-]$

$\mathrm{m}[-]$

$\mathrm{m}[-]$

Figure 13. Transmittance in relation to the distance for different particle sizes and thicknesses, illuminated with a collimated beam.

Figure 13, large particles facilitate a higher transmission at this position. Nevertheless, with increasing distance, the minimum RI ratio $\mathrm{m}$ increases faster for composites with larger particles. It exceeds the small particles' minimum RI ratio at distances $>6 \mathrm{~mm}$. The higher direct transmission of smaller particles allows a value of $m$ that is farther from the value of one (corresponding to a matching $\mathrm{RI}$ ) in order to achieve a transmission $\geq 90 \%$ at large distances. However, the minimum permissible RI ratio is very close to one at long distances for all particles.

To better understand the transmission behavior described above, the angle-dependent transmission of two particle sizes was analyzed in relation to the RI ratio $\mathrm{m}$, Figure 15 . The different curves represent different angles. At each angle, the intensity was evaluated in relation to $\mathrm{m}$. As a result, larger particles exhibit a narrower transmission curve at $\alpha=0^{\circ}$ over $\mathrm{m}$ (red 

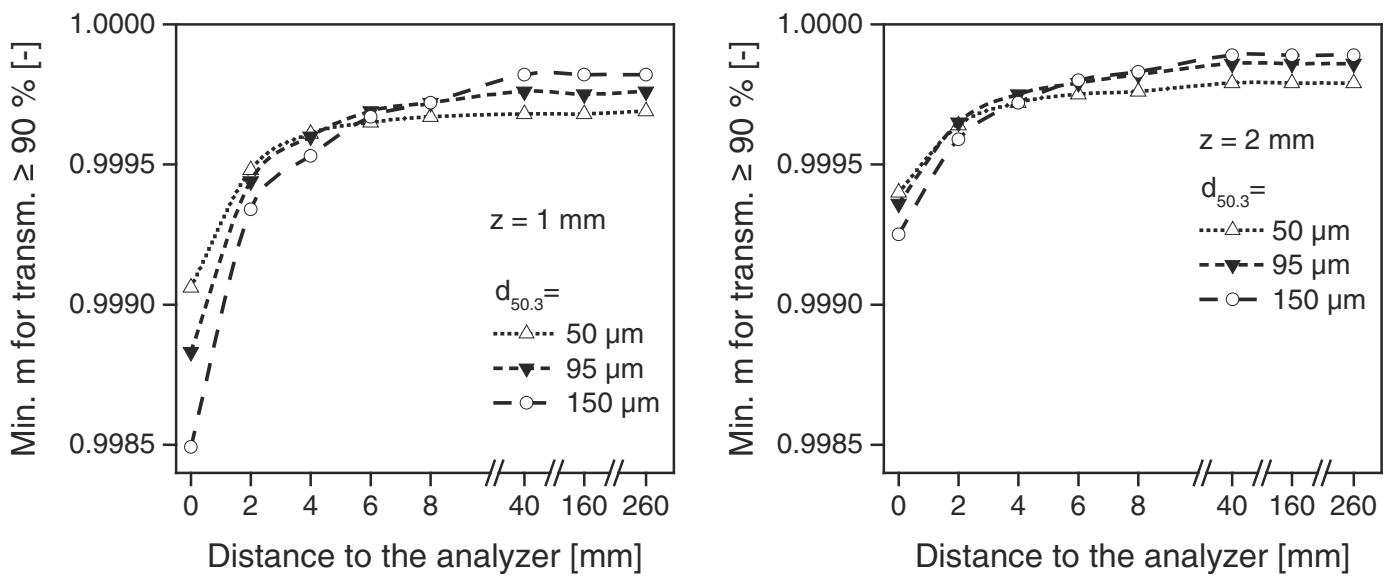

Figure I4. Minimum RI ratio $\mathrm{m}$ for a transmission $\geq 90 \%$ in relation to the distance, particle size and thickness, illumination with a collimated light beam.
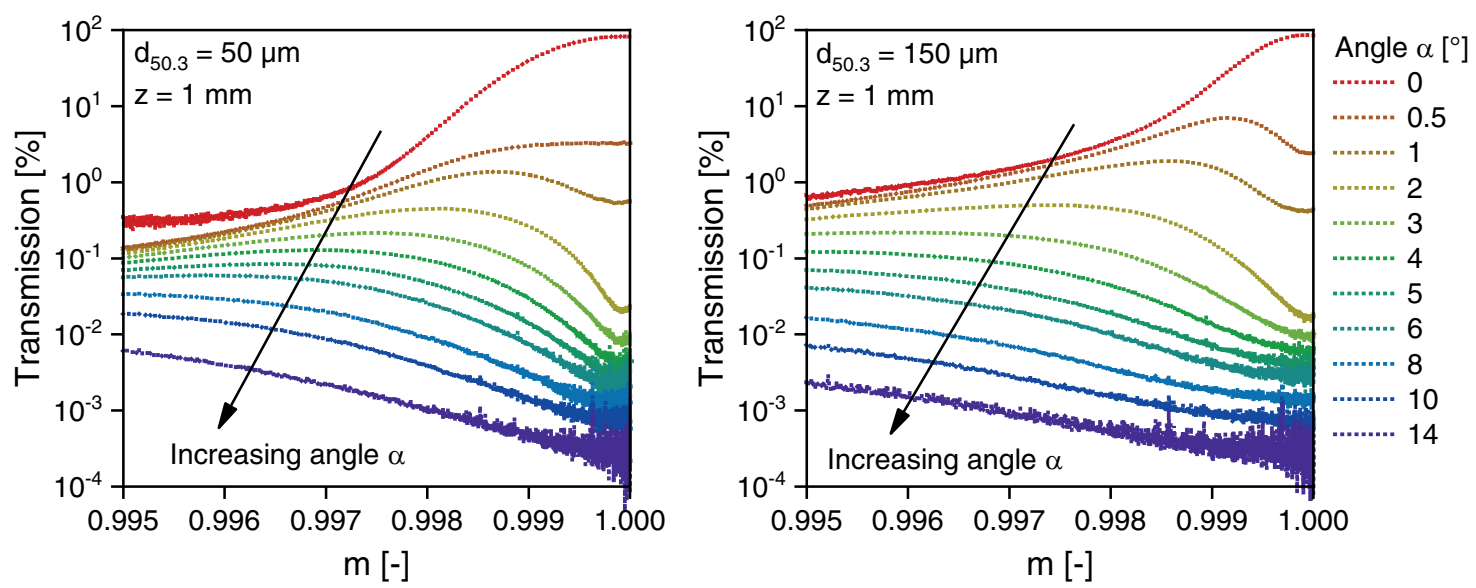

Figure I5. Wavelength dependent scattering for two different particle sizes. The intensity was measured at different angles in relation to the transmission without a specimen at $0^{\circ}$.

dotted line). Figure 13 shows this also at long distances. Figure 15 reveals that larger particles scatter more light at small angles. The majorities of the curves of $0.5^{\circ}$ and $1^{\circ}$ with larger particles (right) are higher than the same curves with smaller particles (left). At large angles, in turn, the light intensity of the small particles exceeds the large ones.

Figure 16 displays the analyzed intensity (left) and the integrated intensity (right) for two different particle sizes at a specific RI ratio $m=0.9985$. The results confirm the outcomes described above. Small particles have a higher direct transmission $\left(\alpha=0^{\circ}\right.$, Figure 16 left $)$. When additional, in small angles scattered light, contributes to the measurement, the transmission of large particles exceeds the transmission of the small ones (Figure 16, right). This behavior applies to standard spectrometer measurements and consequently leads to deceptively higher direct transmission values of larger particles.

Figure 17 shows the light spot, photographed on the image sensor of a camera. The pictures include the brightness curve along the horizontal centerline. Without a specimen in the light path, the white spot is imaged with comparatively sharp edges. When a specimen is inserted into the light path, the light spot appears in red. This is the color of the wavelength with matching RIs at about $640 \mathrm{~nm}$. Large particles cause more blurred edges of the light spot as they scatter more light at small angles. This leads to a brighter background near the spot that reduces the image contrast.

Figure 18 displays the Weber contrast of the spot in comparison to the near background. All specimens diminish the contrast due to the reduced brightness of 

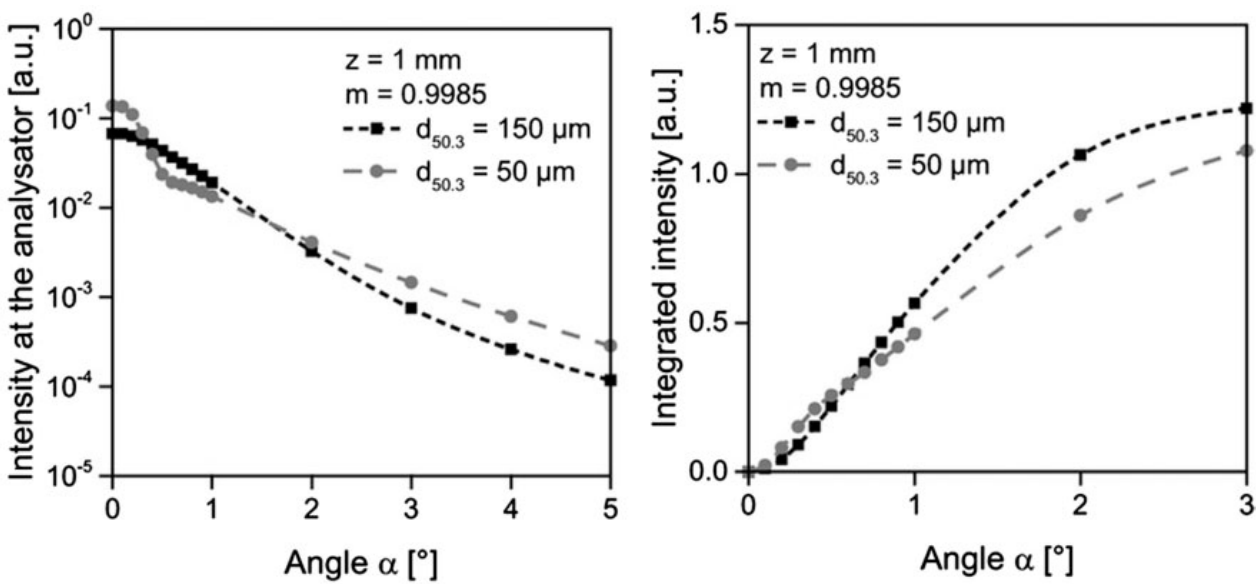

Figure 16. Left: intensity in relation to the angle of the incoming collimated beam; right: integrated intensity for an RI quotient of 0.9985 .

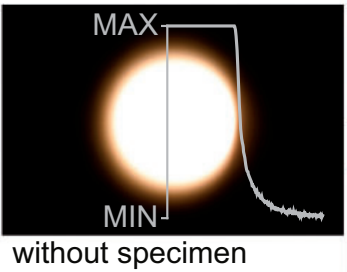

$5 \mathrm{~mm}$
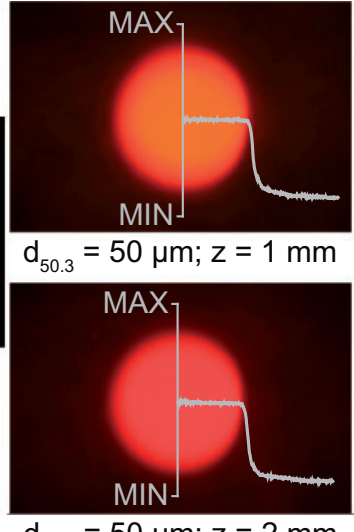

$\mathrm{d}_{50.3}=50 \mu \mathrm{m} ; \mathrm{z}=2 \mathrm{~mm}$

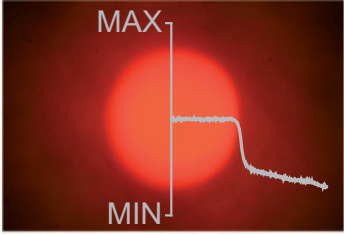

$\mathrm{d}_{50.3}=95 \mu \mathrm{m} ; \mathrm{z}=1 \mathrm{~mm}$

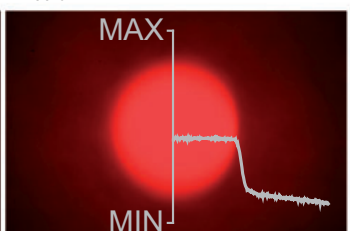

$\mathrm{MIN}$

$\mathrm{d}_{50.3}=95 \mu \mathrm{m} ; \mathrm{z}=2 \mathrm{~mm}$
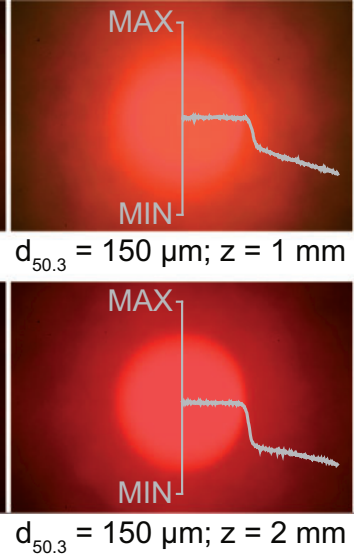

Figure 17. Scattering of a light spot in a distance of $900 \mathrm{~mm}$ with evaluation of the brightness along the horizontal axis; photographed on the image sensor of a camera.

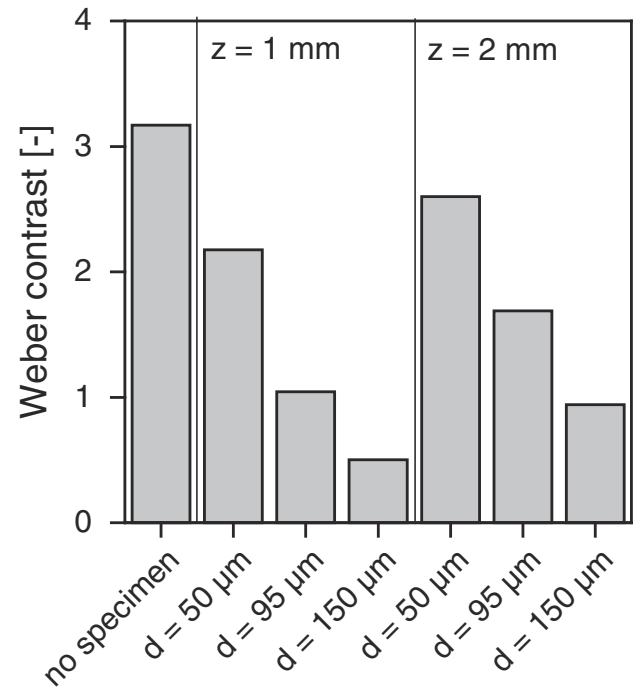

Figure 18. Weber contrast of the specimens when illuminated with a collimated beam. the spot. Additionally, the scattered light leads to a brighter background. The contrast of small particles significantly exceeds the larger particles' contrast. Smaller particles have a higher direct transmission and scatter less light in small angles as described above. As a result, their scattered light is less concentrated in the area of the light spot and results in a darker background.

Figure 19 displays the comparison of the spectrometer measurements with calculations according to Mie's theory. In standard spectrometer measurements, the transmittance is comparable high. In addition, larger particles transmit more light, because significant shares of scattered light contribute to the analyzed transmission. The Mie calculations show a transmittance even below the measurements with the custom spectrometer and smaller particles increase the transmittance. Several reasons may cause this discrepancy: The crushed particles have a randomly rough 


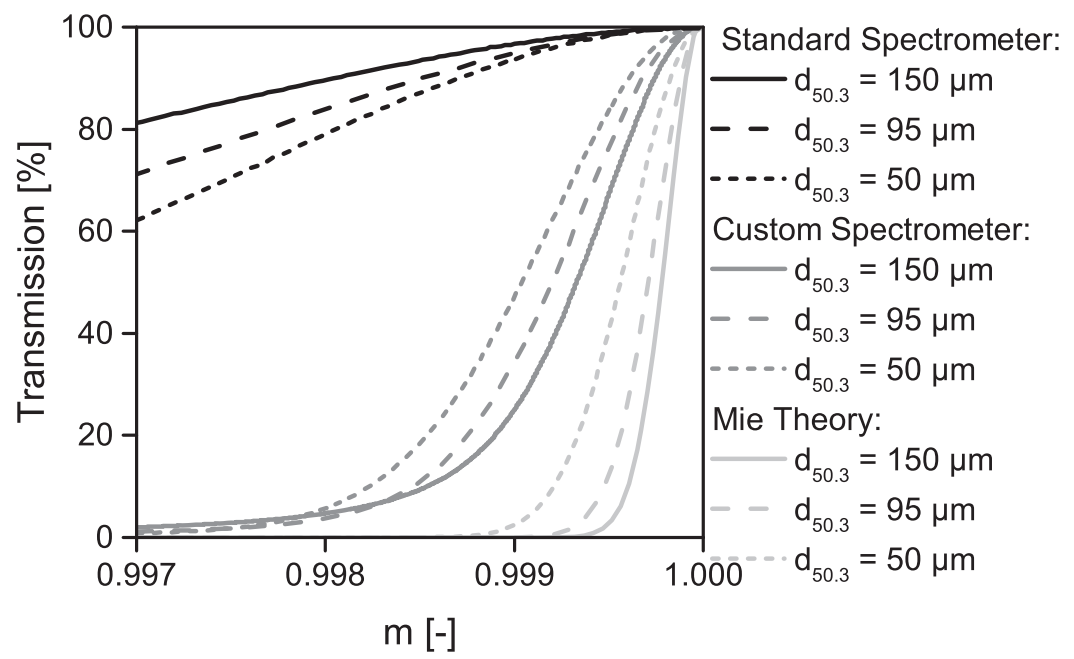

Figure 19. Comparison between different measurements and calculation: standard spectrometer, custom spectrometer in a distance of $220 \mathrm{~mm}$ and calculations according to Mie's theory.
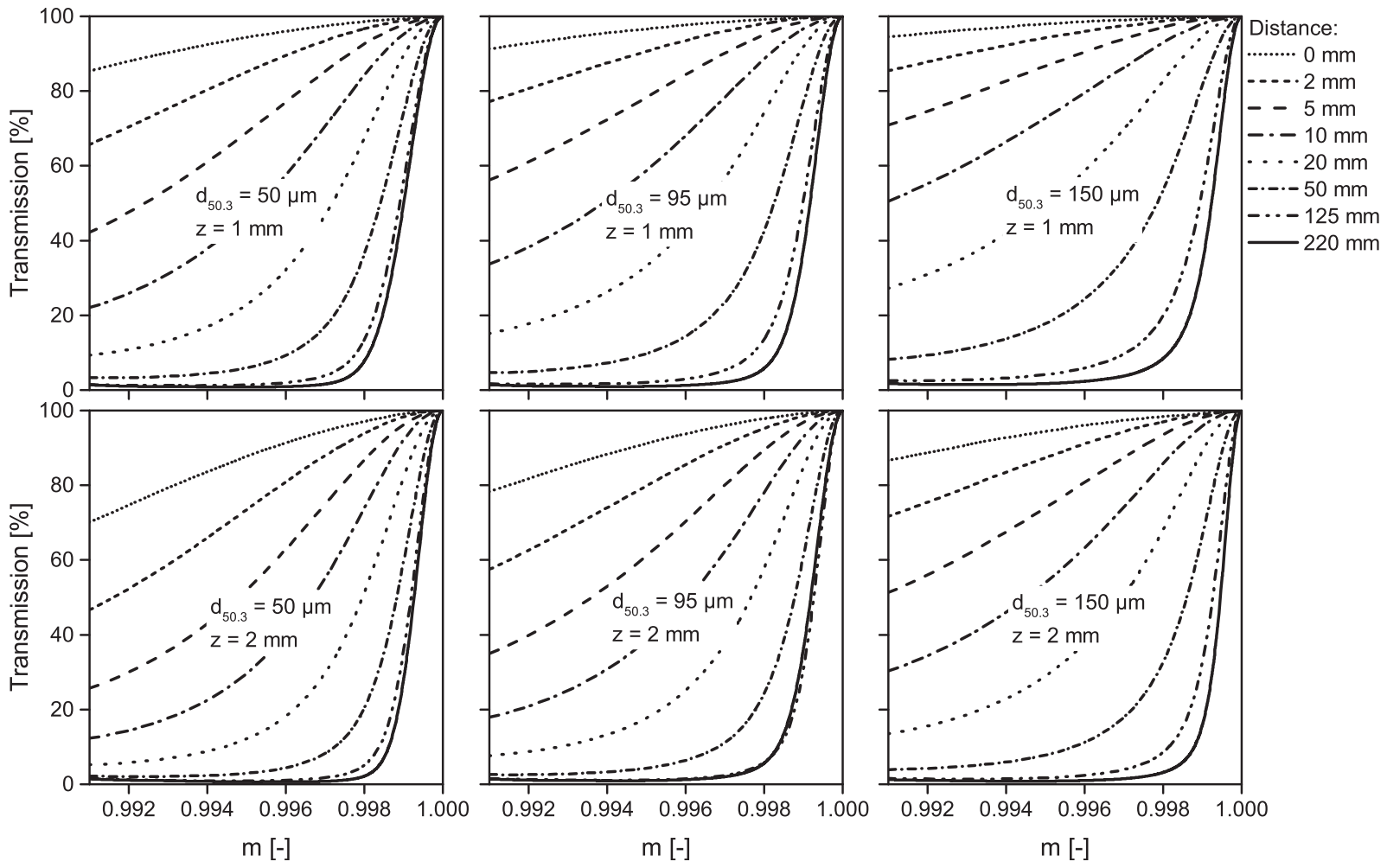

Figure 20. Normalized transmission through a composite, which is placed in front of a diffuse light source.

surface including structures of similar dimensions to the wavelengths of the light. Furthermore, the calculations ignore multiple scattering effects. Dunlap and Howe ${ }^{21}$ demonstrate that packed particles show a significantly broader transmission curve due to interparticle interference.
In comparison to the illumination with collimated light, the scattering behavior of the composite after a diffuse light source was analyzed, Figure 20. This kind of light source behaves similarly to an observed white object behind the composite, since most surfaces exhibit diffuse reflection. As previously described (Figure 5), 
the distance in Figure 20 resembles the space between light source and specimens. The results show that the particles in the composite only show little scattering if the specimen is directly placed on the light source. Furthermore, large particles cause less scattering at this position than small particles. With increasing distance, the scattering of larger particles increases more strongly. Consequently, large particles scatter more at long distances.

The results of Figure 20 are combined in Figure 21, which shows the minimum allowed RI ratio $\mathrm{m}$ for a transmission $\geq 90 \%$. A low RI ratio of 0.987 is possible for large particles when the specimen is placed directly on the light source. Nevertheless, the minimum allowed value for $\mathrm{m}$ increases rapidly with longer distances. According to the measurements with a collimated light beam, large particles transmit more light at short distances and less light at long distances.

Potential applications for glass-particles reinforced materials that require high transmission over a wide wavelengths range, are glass-reinforced plastic glazing or housings. Glass as filler can enhance stiffness and strength. Another potential application is polymer overmoulding composites. Glass particles lead to reduced shrinkage and thermal expansion, as described in the introduction section. If the object is positioned directly behind the composite, as with overmoulded parts, large particles transmit much light over a broad range of wavelengths, even at very high filling degrees. Conversely, reinforced glazing, which must provide a clear view of an object in the distance, has the high requirement of having very small RI differences. Nevertheless, small particles are more suitable due to their higher direct transmittance. Additionally, small particles scatter less light at small angles. This scattered light affects the clear visibility of detailed objects comparatively strong. ${ }^{10}$ This becomes clear by the appearance of the diffuse light source behind the specimens in Figure 22.

If the specimen is placed directly in front of the diffuse light source, the spot is visually hardly affected. Only the $2 \mathrm{~mm}$ thick specimens with small and medium particles blur the edges slightly due to scattered blue light. Blue is the color of the visible wavelength with the highest RI difference of the analyzed material combination. The scattering becomes significantly

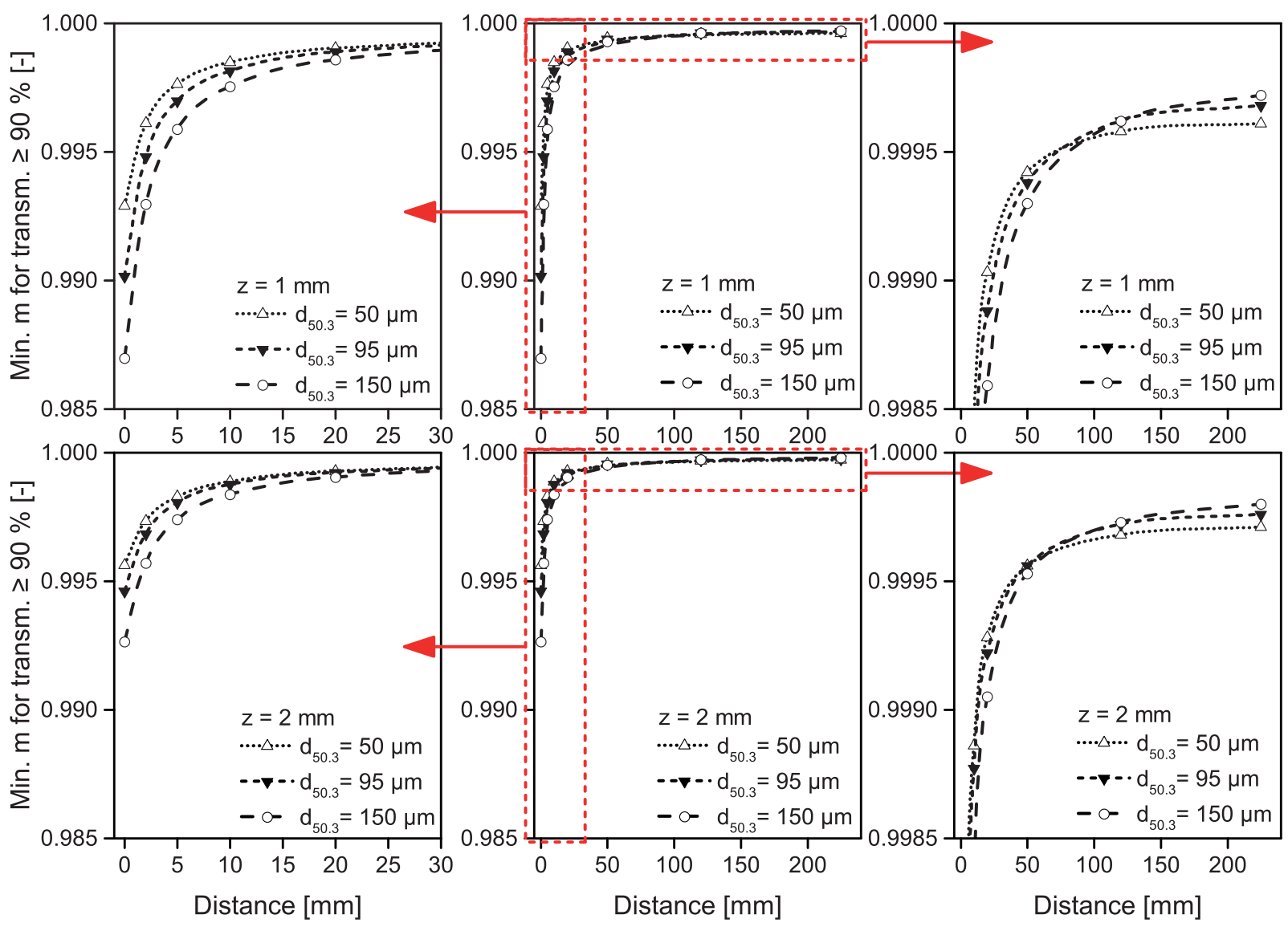

Figure $\mathbf{2} \mathbf{I}$. Minimum allowed $\mathrm{RI}$ ratio $\mathrm{m}$ to achieve a transmission of min. Ninety percent for the specimens with a thickness of $\mathrm{I} m \mathrm{~m}$ (above) and $2 \mathrm{~mm}$ (below) in front of a diffuse light source. 

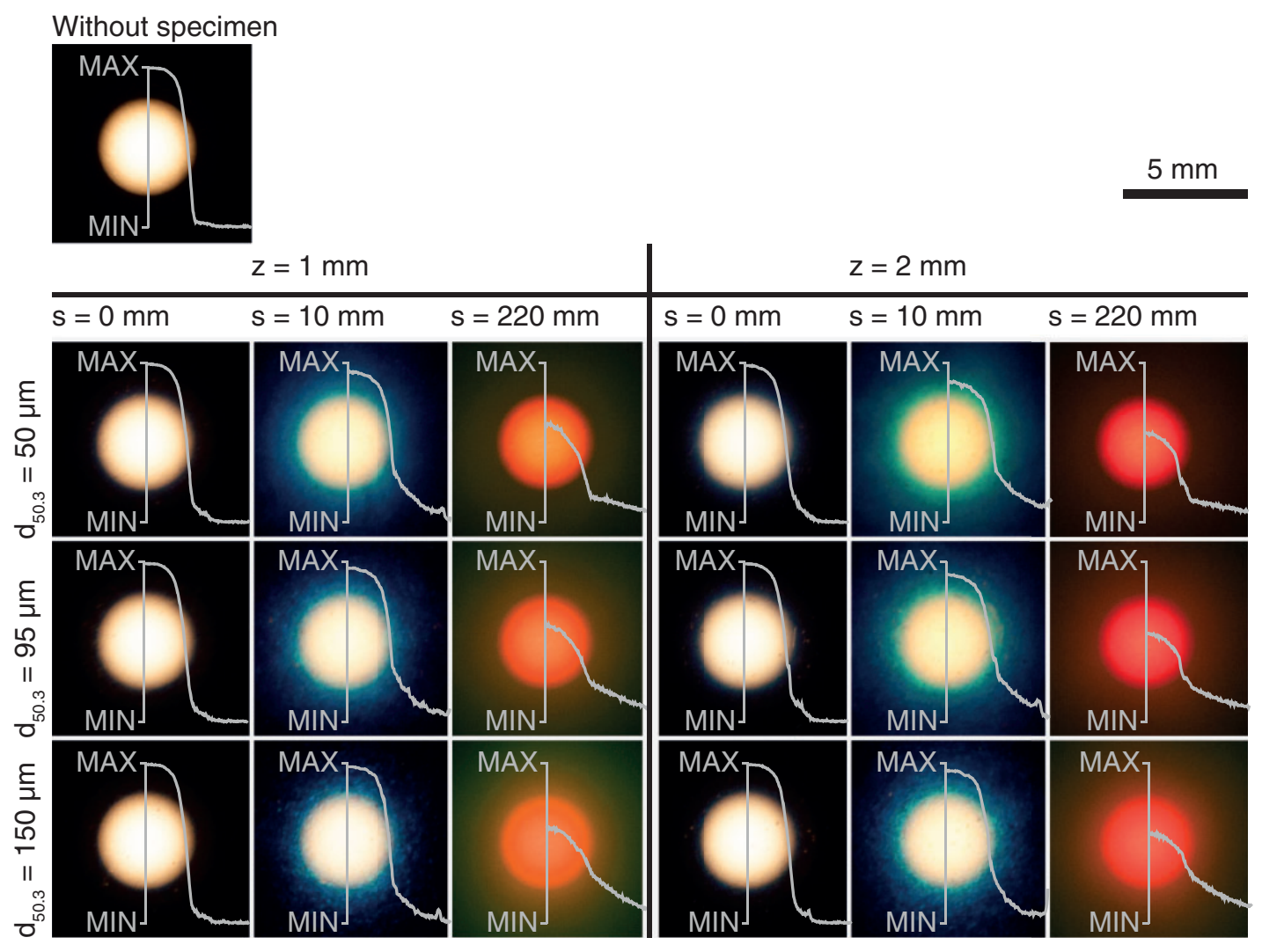

Figure 22. Appearance of a white diffuse light source behind the specimens with evaluation of the luminescence along the horizontal axis; $\mathrm{s}=$ distance between light source and specimen.

stronger when the specimen is inserted $10 \mathrm{~mm}$ in front of the light source. Here too, large particles improve the visibility of the light spot's contours. However, if the distance between the light source and the specimens is $220 \mathrm{~mm}$, the spot appears in red light. This is the color of the wavelength of about $640 \mathrm{~nm}$ with a matching RI. Small particles now improve the visibility of the spot because the scattered light is more uniformly dispersed in the background and less concentrated in the area of the spot.

Figure 23 confirms these results by the evaluation of the specimens' Weber contrast. Even if the specimens are placed directly behind the composite, they reduce the contrast to $50 \%$, although this reduction is hardly visible on the photographs in Figure 22. At this position and at a distance of $10 \mathrm{~mm}$ from the light source, large particles lead to a better contrast because of stronger scattering at very small angles. This keeps the light close to the spot at short distances. Contrary, at a long distance of $220 \mathrm{~mm}$, the contrast of small particles is twice as large as the contrast of large particles. However, the contrast in this position is generally at a low level.

Another picture emerges though, if the light source has a specific wavelength (here: $550 \mathrm{~nm}$ ), which is not

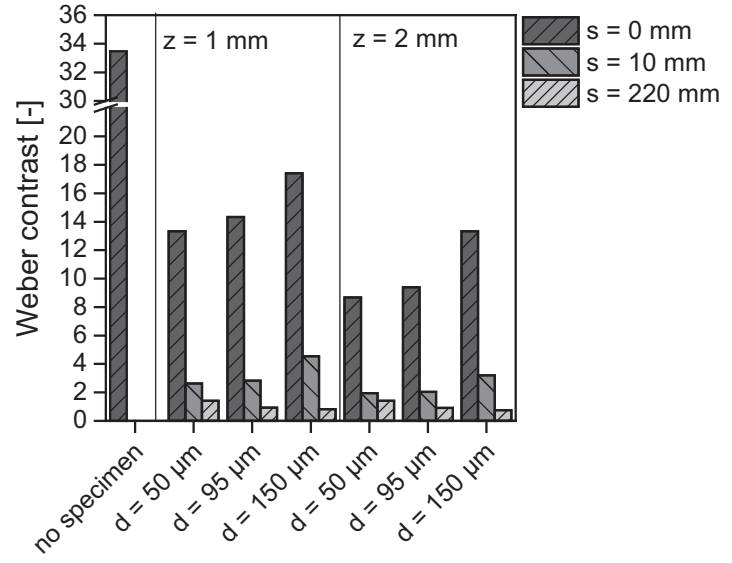

Figure 23. Weber contrast of the diffuse light source behind the specimens.

the wavelength with a matching RI (Figure 24). The contour of the spot is clearly visible only if the specimen is placed directly in front of the light source. With increasing distance and thickness as well as deceasing particle size, the spot becomes blurred and vanishes completely with larger distance. Due to the RI quotient $\mathrm{m}$ of about 0.9975 at $550 \mathrm{~nm}$, almost no light is recorded by the camera at a distance of $220 \mathrm{~nm}$. 


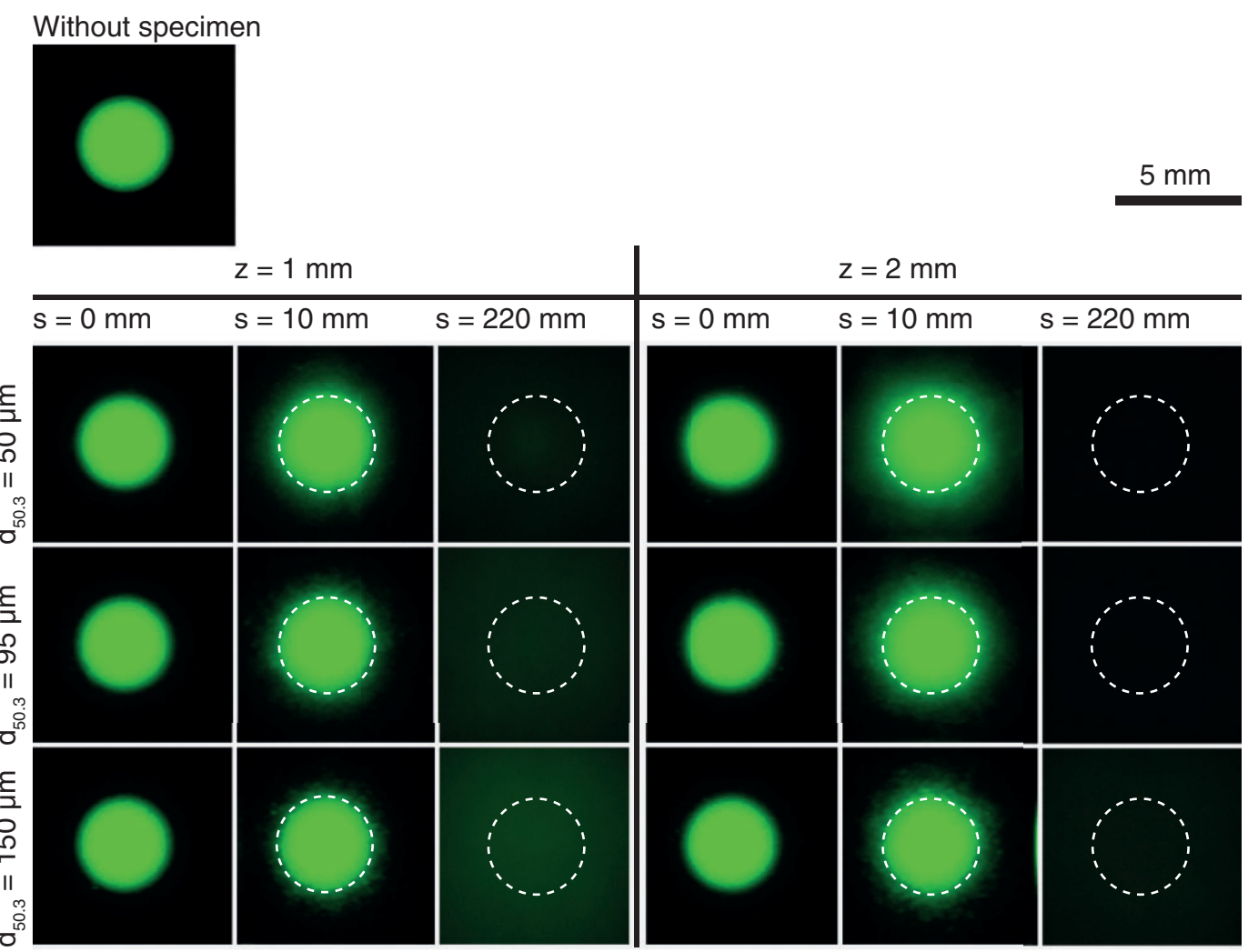

Figure 24. Appearance of a green $(550 \mathrm{~nm})$ light source behind the specimens. $\mathrm{s}=$ Distance between light source and specimen; the white dashed circles at distances of $10 \mathrm{~mm}$ and $220 \mathrm{~mm}$ mark the position of the light source without a specimen.

\section{Conclusion}

The results show that a standard spectrometer is not sufficient to analyze the light transmission and scattering properties of transparent composites with a very similar RI between filler particles and matrix. In addition to directly transmitted light, standard spectrometers also collect significant shares of scattered light. This leads to an apparently higher transmission of composites with larger particles. However, intensity measurements with relation to the angle reveal that small glass particles transmit more light directly, while larger particles scatter more light at small angles. The evaluation of photographs of a white light source shows that the scattering in small angles leads, in the case of long distances between light source and specimen, to a more blurred image of the light source.

The application-oriented measurements with a diffuse light source reveal the potential of such a material combination, as it is placed directly on an object or on a diffuse light source. High filling degrees and RI differences are possible without visually affecting the transmission or visibility of an object behind the composite. In this setup, large particles have the advantage of imaging the light source with higher contrast due to their scattering properties. If the distance increases, the contour of the diffuse light source is still well visible, but it is displayed in the color of the wavelength with matching RIs. Small particles then lead to a better contrast because their scattered light is less concentrated in the area of the light spot. Rather, it is more uniformly dispersed in the background. However, if the diffuse light source behind the composite has a specific wavelength (as when an object has a specific color), or if the matching RI is located at a wavelength outside the visual range, the image blurs strongly with increasing distance.

\section{Declaration of Conflicting Interests}

The author(s) declared no potential conflicts of interest with respect to the research, authorship, and/or publication of this article.

\section{Funding}

The author(s) disclosed receipt of the following financial support for the research, authorship, and/or publication of this article: The authors would like to acknowledge the funding of the Deutsche Forschungsgemeinschaft (DFG) through the Cluster of Excellence Engineering of Advanced Materials.

\section{ORCID iD}

Wolfgang Wildner (D) http://orcid.org/0000-0002-5251-8720. 


\section{References}

1. Hashemi S, Din KJ and Low P. Fracture behavior of glass bead-filled poly(oxymethylene) injection moldings. Polym Eng Sci 1996; 36: 1807-1820.

2. Lee $\mathbf{J}$ and Yee AF. Fracture of glass bead/epoxy composites: on micro-mechanical deformations. Polymer 2000; 41: 8363-8373.

3. Wildner W and Drummer D. The mechanical and optical properties of injection-moulded PMMA, filled with glass particles of a matching refractive index. Polym Polym Compos 2017; 25: 419-427.

4. Naganuma T, Iba H and Kagawa Y. Optothermal properties of glass particle-dispersed epoxy matrix composite. J Mater Sci Lett 1999; 18: 1587-1589.

5. Kagawa Y, Iba H, Tanaka M, et al. Fabrication and optical/thermal properties of glass particle-epoxy optically transparent composites. Acta Mater 1998; 46: 265-271.

6. Ajayan PM, Schadler LS and Braun PV. Nanocomposite science and technology. Weinheim, Germany: WileyVCH, 2003.

7. Wildner W and Drummer D. A fiber optic temperature sensor based on the combination of epoxy and glass particles with different thermo-optic coefficients. Photonic Sens 2016; 6: 295-302.

8. Wildner W and Drummer D. A fiber optic temperature sensor based on the combination of two materials with different thermo-optic coefficients. IEEE Sens $J$ 2015; 16 : 688-692.

9. van de Hulst HC. Light scattering by small particles. New York: Dover Publications, 1981.

10. Meeten GH. Optical properties of polymers. England: Elsevier Applied Science Publishers, 1986.

11. Groever B, Heshmat B and Raskar R. Tyndall windows: tunable scattering of disordered solid-liquid matching mixtures. ACS Photonics 2016; 3: 930-935.

12. Kahnert M, Nousiainen T, Thomas MA, et al. Light scattering by particles with small-scale surface roughness: comparison of four classes of model geometries. J Quant Spectrosc Radiat Transf 2012; 113: 2356-2367.

13. Muinonen K, Nousiainen T, Lindqvist H, et al. Light scattering by Gaussian particles with internal inclusions and roughened surfaces using ray optics. J Quant Spectrosc Radiat Transf 2009; 110: 1628-1639.

14. Zubko E, Muinonen K, Shkuratov Y, et al. Scattering of light by roughened Gaussian random particles. J Quant Spectrosc Radiat Transf 2007; 106: 604-615.

15. Christiansen C. Untersuchungen über die optischen Eigenschaften von fein vertheilten Körpern. Annalen der Physik 1884; 259: 298-306.

16. Christiansen C. Untersuchungen über die optischen Eigenschaften von fein vertheilten Körpern. Annalen der Physik 1885; 260: 439-446.

17. Naganuma $T$ and Kagawa $Y$. Effect of particle size on light transmittance of glass particle dispersed epoxy matrix optical composites. Acta Mater 1999; 47: 4321-4327.
18. Iba H, Chang T and Kagawa Y. Optically transparent continuous glass fibre-reinforced epoxy matrix composite: fabriction, optical and mechanical properties. Compos Sci Technol 2002; 62: 2043-2052.

19. Naganuma T and Kagawa Y. Effect of total particle surface area on the light transmittance of glass particle-dispersed epoxy matrix optical composites. J Mater Res 2002; 17: 3237-3241.

20. Sato H, Iba H, Naganuma T, et al. Effects of the difference between the refractive indices of constituent materials on the light transmittance of glass-particle-dispersed epoxy-matrix optical composites. Philos Mag B Phys Condens Matter Statistical Mech Electron Opt Magn Prop 2002; 82: 1369-1386.

21. Dunlap PN and Howe SE. Design of particulate composites for optical applications. Polym Compos 1991; 12: 39-47.

22. Jennings BR and Jerrard HG. Rayleigh-Gans-Debye and Mie theories in the determination of spherical particle sizes. J Colloid Sci 1965; 20: 448-452.

23. Lin H, Day DE and Stoffer JO. Model for the temperature dependent transmission of optically transparent poly(methyl methacrylate) composites. J Mater Res 1993; 8: 364-370.

24. Olson JR, Day DE and Stoffer JO. Fabrication and mechanical properties of an optically transparent glass fiber/polymer matrix composite. J Compos Mater 1992; 26: 1181-1192.

25. Weaver KD and Stoffer JO. Preparation and properties of optically transparent, pressure-cured poly(methyl methacrylate) composites. Polym Compos 1993; 14: 515-523.

26. Lin H, Day DE, Weaver KD, et al. Temperature and wavelength dependent transmission of optically transparent glass fibre poly (methyl methacrylate) composites. J Mater Sci 1994; 29: 5193-5198.

27. Kang S, Lin H, Day DE, et al. Optically transparent polymethyl methacrylate composites made with glass fibers of varying refractive index. J Mater Res 1997; 12: 1091-1101.

28. Iba $\mathrm{H}$ and Kagawa $\mathrm{Y}$. Light transmittance of continuous fibre-reinforced composites: analysis, model experiment and parametric study. Philos Mag B Phys Condens Matter Statistical Mech Electron Opt Magn Prop 1998; 78: 37-52.

29. Khanarian G and Walpita LM, Transparent polymer composites having a low thermal expansion coefficient. Patent US005783624A, 1998.

30. Shibahara S, Oka W, Shimobe Y, Kuramoto H and Nakao T, Transparent composite composition. Patent US20050203239A1, 2005.

31. Kato $\mathrm{T}$ and Sugawara T, Glass filler for transparent resin. Patent application JP2001261367A, 2001.

32. Kushida T, Nakasuji I and Toyama T. Epoxy resin composition for sealing optical semiconductor and optical semiconductor device. Patent application JP2003003043A, 2003.

33. Meixner L, Meyers D, Morris M, et al. Filled epoxy resin composition and its production method. Patent JP4570547B2, 2010. 
34. Ito $\mathrm{H}$ and Ota S. Epoxy resin composition for optical semiconductor element sealing and optical semiconductor apparatus using the same. Patent application JP2005298701A, 2005.

35. Shimada K and Yamada J. Epoxy resin for sealing optical semiconductor element and optical semiconductor device. Patent application JP2001261933A, 2001.

36. Okamoto M and Chiba J, Polycarbonate resin composition and process for producing the same. Patent US5510414A, 1996.

37. Shibahara S, Shimobe Y and Kuramoto H, Transparent composite composition Patent US20040126592A1, 2003.

38. Iwakiri $\mathrm{T}$ and Imaizumi $\mathrm{H}$, Transparent rigid resin molded product and process for producing the same. Patent US005733659A, 1998.

39. Shunsaku Mino S, Morohashi Y, Tanikoshi T, et al. Plastic material with dimming performance. Patent application JPS5949245A, 1984.

40. Wilson PS, Window for motor vehicle. Patent US6365277B1, 2002.
41. A.KRÜSS Optronic GmbH. Engineering Drawing for the Measurement Prism, 2016.

42. Bliedtner $\mathbf{J}$ and Gräfe G. Optiktechnologie, 2nd ed. München, Germany: Hanser Verlag, 2010.

43. Rheims J, Köser J and Wriedt T. Refractive-index measurements in the near-IR using an Abbe refractometer. Meas Sci Technol 1997; 8: 601-605.

44. Kedenburg S, Vieweg M, Gissibl T, et al. Linear refractive index and absorption measurements of nonlinear optical liquids in the visible and near-infrared spectral region. Opt Mater Express 2012; 2: 1588-1611.

45. Jenkins F and White H. LSC fundamentals of optics. New York: McGraw-Hill Education, 2001.

46. Laven P. MiePlot. www.philiplaven.com/mieplot.htm, (2017, accessed 20 October 2016).

47. Li WB, Lu CH and Zhang JC. A lower envelope Weber contrast detection algorithm for steel bar surface pit defects. Opt Laser Technol 2013; 45: 654-659. 